\title{
Diagnostic value of chest computed tomography imaging for COVID-19 based on reverse transcription-polymerase chain reaction: a meta-analysis
}

\author{
Jing Liu' ${ }^{1}$ Xue Yang ${ }^{2}$, Yunxian Zhu' ${ }^{1}$ Yi Zhu' ${ }^{1}$, Jingzhe Liu ${ }^{3}$, Xiantao Zeng ${ }^{4}$ and Hongjun Li $^{*}$
}

\begin{abstract}
Background: The computed tomography (CT) diagnostic value of COVID-19 is controversial. We summarized the value of chest CT in the diagnosis of COVID-19 through a meta-analysis based on the reference standard.

Methods: All Chinese and English studies related to the diagnostic value of CT for COVID-19 across multiple publication platforms, was searched for and collected. Studies quality evaluation and plotting the risk of bias were estimated. A heterogeneity test and meta-analysis, including plotting sensitivity (Sen), specificity (Spe) forest plots, pooled positive likelihood ratio (+LR), negative likelihood ratio (-LR), dignostic odds ratio (DOR) values and 95\% confidence interval (CI), were estimated. If there was a threshold effect, summary receiver operating characteristic curves (SROC) was further plotted. Pooled area under the receiver operating characteristic curve (AUROC) and 95\% Cl were also calculated.
\end{abstract}

Results: Twenty diagnostic studies that represented a total of 9004 patients were included from 20 pieces of literatures after assessing all the aggregated studies. The reason for heterogeneity was caused by the threshold effect, so the AUROC $=0.91$ (95\% Cl: 0.89-0.94) for chest CT of COVID-19. Pooled sensitivity, specificity, +LR, -LR from 20 studies were 0.91 (95\% Cl: 0.88-0.94), 0.71 (95\% Cl: 0.59-0.80), 3.1 (95\% Cl: 2.2-4.4), 0.12 (95\% Cl: 0.09-0.17), separately. The $P^{2}$ was $85.6 \%(P=0.001)$ by Q-test.

Conclusions: The results of this study showed that CT diagnosis of COVID-19 was close to the reference standard. The diagnostic value of chest CT may be further enhanced if there is a unified COVID-19 diagnostic standard. However, please pay attention to rational use of $\mathrm{CT}$.

Keywords: COVID-19, Tomography, X-ray computed, Reference standard

\section{Background}

Coronavirus disease 2019 (COVID-19) is an acute infectious disease caused by severe acute respiratory syndrome coronavirus 2 (SARS-CoV-2) and is primarily

*Correspondence: lihongjun00113@ccmu.edu.cn

2 Department of Radiology, Beijing Youan Hospital Capital Medical University, Beijing 100069, People's Republic of China

Full list of author information is available at the end of the article characterized by pulmonary inflammatory lesions. It can also cause damage to the digestive system [1-3], nervous system $[4,5]$, urinary system [6] and other systemic organs, with corresponding clinical symptoms. COVID19 was identified as the health emergency of national concern by World Health Organization on 30 January, 2020 since its initial detection in December 2019 [7]. COVID-19 has highly transmissible [8]. Current clinical 
studies show that there are no specific therapeutic drugs for treatment [9].

The reference standard for diagnosing COVID-19 is reverse transcription-polymerase chain reaction (RTPCR) [10]; however, it suffers from numerous flaws, such as poor sensitivity [11], producing false negatives, etc., all of which have also been noted in the studies $[12,13]$. A study indicated that RT-PCR was not a perfect reference standard [14]. What's more, a succession of countries [15] had reported the discovery of COVID-19 variant strains, and the proportion is increasing. The most feared of the COVID-19 variants could accelerate the spread of the disease. Researchers at Vita's laboratory in Helsinki, Finland considered that RT-PCR may not be able to detect the mutated virus (http://www.zhihu.com/question/ 445217928). Feng et al. pointed out that the abundance of genomic data made it possible to reassess the applicability of RT-PCR to ensure that it was applicable to mutant strain [16].

Computed tomography (CT) played an important role in the global fight against COVID-19 [11]. Some studies have shown that CT can be used as a primary auxiliary screening and diagnostic tool in pandemic areas [17-19]. The COVID-19 pandemic has put enormous pressure on global health resources, which creates an urgent need for researchers to find a reasonable diagnosis. In this metaanalysis, we aim to summarize the diagnostic value and existing problems of CT.

\section{Methods}

\section{Search strategy}

We searched for published studies in English and Chinese as well as what could be considered gray area studies in PubMed, Embase, Web of Science, Cochrane Library, CBM (http://sinomed.ac.cn/), CNKI (https:// www.cnki.net/), Wan-fang (https://www.wanfangdata. com.cn/index.html), and VIP (http://cstj.cqvip.com/) databases between 1 December, 2019 and 16 August, 2021. The search strategy was developed under the guidance of professionals and involved matching subject terms with free words. The PubMed search strategy was ((“"COVID-19”[Mesh]) OR $((()((()((2019$ novel coronavirus disease[Title/Abstract]) OR (COVID19[Title/ Abstract])) OR (COVID-19 pandemic[Title/Abstract])) OR (SARS-CoV-2 infection[Title/Abstract])) OR (COVID-19 virus disease[Title/Abstract])) OR (2019 novel coronavirus infection[Title/Abstract])) OR (2019$\mathrm{nCoV}$ infection[Title/Abstract])) OR (coronavirus disease 2019[Title/Abstract])) OR (coronavirus disease-19[Title/ Abstract])) OR (2019-nCoV disease[Title/Abstract])) OR (COVID-19 virus infection[Title/Abstract]))) AND (“"Tomography, X-Ray Computed”[Mesh]) OR
(((((X-Ray Comput* Tomography[Title/Abstract]) OR (CT[Title/Abstract])) OR (comput* tomograph*[Title/ Abstract])) OR (CT Scan*[Title/Abstract])) OR (Helical CT*[Title/Abstract])) OR (CT X Ray*[Title/Abstract])))) AND (sensitiv*[Title/Abstract] OR sensitivity and specificity[MeSH Terms] OR (predictive[Title/Abstract] AND value*[Title/Abstract]) OR predictive value of tests[MeSH Term] OR accuracy*[Title/Abstract]). We prospectively submitted the meta-analysis protocol for registration of PROSPERO (CRD42020225262).

\section{Selection criteria}

Inclusion criterias were as follow-up: (1) retrospective or prospective diagnostic test study; (2) collect clinical history, suspected COVID-19, confirmed COVID-19, and chest CT results of diagnosed/suspected COVID-19; (3) COVID-19 confirmed by the reference standard RT-PCR; (4) direct or indirect information sufficient to extract $2 \times 2$ table data for diagnosis of COVID- 19 by chest CT, including true positive (TP), false positive (FP), false negative (FN) and true negative (TN).

Literatures were excluded if they were (1) duplicates, case reports, reviews, systematic reviews, meta-analyses, conference abstracts, letters, non-original studies, and unrelated studies; (2) failure to extract the $2 \times 2$ table data; (3) the full text was unavailable; (4) not confirmed by the reference standard.

\section{Data extraction and quality assessment}

Two authors independently screened the studies for quality assessment and data extraction according to the inclusion and exclusion criteria, and disagreements were resolved by reaching a consensus or consulting a third senior specialist. The extracted data included: (1) First author, study site, study time, publication year, age, gender, sample size, study type (prospective, retrospective), and case selection; (2) Diagnostic reference standard, i.e., RT-PCR; (3) Outcome indicators included the sensitivity (Sen), specificity (Spe), accuracy (Ac), negative predictive value (NPV), positive predictive value (PPV), TP, FP, $\mathrm{FN}$, and $\mathrm{TN}$ of diagnosis by chest $\mathrm{CT}$, which were used to build the $2 \times 2$ table.

A quality evaluation was done in the diagnostic test of Review Manager 5.3 (the Cochrane Collaboration, London, UK), and for the requirements of Quality Assessment of Diagnostic Studies-2 (QUADAS-2), the evaluation rules applicable to this study were developed by referring to relevant studies [20]. On this basis, if the opinions of the two authors were still not consistent, the evaluation rules would be renegotiated to reach a consensus and further improve and supplement the preestablished evaluation rules. 


\section{Statistical analysis}

Meta-disc1.4.0.0 (Clinical Biostatistics Unit, Madrid, Spain) and Stata14.0 (Computer Resource Center, Texas, US) software were used for statistical analysis. The Q test was utilized to analyze the heterogeneity between the results of each study. If $P<0.01$, the difference was considered statistically significant, and the heterogeneity was quantitatively judged based on $I^{2}$ value. If $I^{2}>50 \%$, heterogeneity was considered significant, and the random effects model was selected. Otherwise, the fixed effects model was selected; Spearman's rank correlation coefficient and summary receiver operating characteristic curve (SROC) between the logarithm of sensitivity and the logarithm of (1-specificity) were used to determine whether there was a threshold effect. Forest plots of Sen and Spe were made to calculate the pooled positive likelihood ratio (+LR), negative likelihood ratio (-LR), dignostic odds ratio (DOR) values and 95\% confidence interval $(C I)$; if there was a threshold effect, SROC was further plotted to calculate the pooled area under the receiver operating characteristic curve (AUROC). Publication bias was assessed with Deeks' plots.

\section{Results}

\section{Literature search}

The flow chart for the screening of included literatures is shown in Fig. 1. According to the search strategy, a total of 6537 pieces of literatures were initially screened; 3330 duplicates were excluded by the management application; 214 pieces of literatures of meta-analysis, systematic review and review were excluded; 134 pieces of literatures of case report were excluded; 1 piece of literature of animal experiment was excluded. In addition, 2791 pieces of literatures with irrelevant content were excluded after the titles and abstracts were reviewed; 42 pieces of literatures from which the $2 \times 2$ table could not be obtained were excluded after a review of the full text; 3 pieces of literatures were error in original data; 2 pieces of literatures without full text. Finally, 20 pieces of literatures were included, which were from China, Italy, France, United Kingdom, the Netherlands, Brazil and India.

\section{Quality evaluation}

The basic characteristics of the included 20 literatures are shown in Table 1 . These literatures were produced by emergency departments or fever clinics in different regions.

The results of QUADAS-2 are shown in Fig. 2. In 9/20 pieces of literatures, the flow and timing were evaluated as high risk because these studies did not include all the cases, which might cause bias. In $8 / 20$ pieces of literatures, the risk of bias with regards to the reference standard was unclear because these studies did not specifically describe whether repeat testing was performed for the first negative RT-PCR test. In 5/20 pieces of literatures, the risk of the index test was unclear because the literatures did not specifically show whether the film was read through the blind method. In 2/20 pieces of literatures, the high risk of patient selection was mainly because all the patients in the studies could be definitively diagnosed by the index test and the lack of patients that were difficult to diagnose. Furthermore, in $2 / 20$ pieces of literatures, the risk of patient selection was unclear because of the absence of explanatory notes confirming the presence of difficult to diagnose patients.

\section{Pooling of effect sizes and plotting}

Pooled sensitivity, specificity, +LR, -LR from 20 studies were 0.91 (95\% CI: 0.88-0.94), 0.71 (95\% CI: 0.590.80), 3.1 (95\% CI: 2.2-4.4), 0.12 (95\% CI: 0.09-0.17), separately. The $I^{2}$ was $85.6 \%(P=0.001)$ by Q-test. The effect size was pooled with the random-effects model. DOR was 25.02 (95\% CI: 16.97-36.89). The forest plots of Sen, Spe and DOR (OR) value of CT in the 20 literatures are shown in Fig. 3. The positive likelihood ratio (+LR) and negative likelihood ratio (-LR), the likelihood ratio scatter plot, the pre-test probability/ post-test probability is shown in Fig. 4. It can be seen that the misdiagnosis rate and missed diagnosis rate of the index test for the included literatures used for this study are small. In this study, the pre-test probability by the physician was $20 \%$, the post-test positive predictive value was $44 \%$, and the post-test negative predictive value was $3 \%$, indicating that the physician's judgment of the patient is important. This also indicates that if the pre-test probability is increased, then the index test can proficiently predict the disease.

\section{Threshold effect analysis}

The Spearman's correlation coefficient between the logarithm of sensitivity and the logarithm of $(1-$ specificity) was determined to be $r=0.681 \quad(P=0.001)$, indicating significant correlation and threshold effect heterogeneity. SROC was plotted, showing a "shoulder-arm"-like change. Therefore, Sen and Spe could not be combined alone in this study. In order to further to reflect the real diagnostic accuracy of CT, AUROC was calculated to be 0.91 (95\% CI: 0.89-0.94). The results (AUROC > 90\%, and $P<0.05)$ indicate that $\mathrm{CT}$ has a diagnostic accuracy close to the reference standard, and it is basically capable of making a correct diagnosis. SROC is shown in Fig. 5.

\section{Sensitivity analysis and Deeks' publication bias test}

It can be clearly seen from Fig. $6 a$ to $d$ that there is strong sensitivity in one original study (No. 14) [34]. 

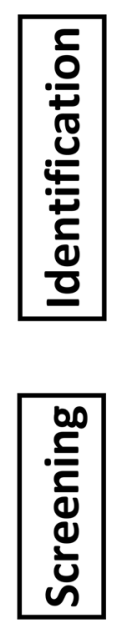

Database Search $n=6537$

Cochrane $=34$ Embase $=1053$ Pubmed $=1468$ Web of Science $=1097$

Wanfang $=874 \quad \mathrm{CBM}=1224 \quad \mathrm{CNKI}=396 \quad \mathrm{VIP}=391$

Duplicate literatures

$n=3330$

Literatures excluded $n=349$

(Meta, systematic reviews, and reviews $n=214$ Case report $n=134$

Animal experiment $n=1$ )

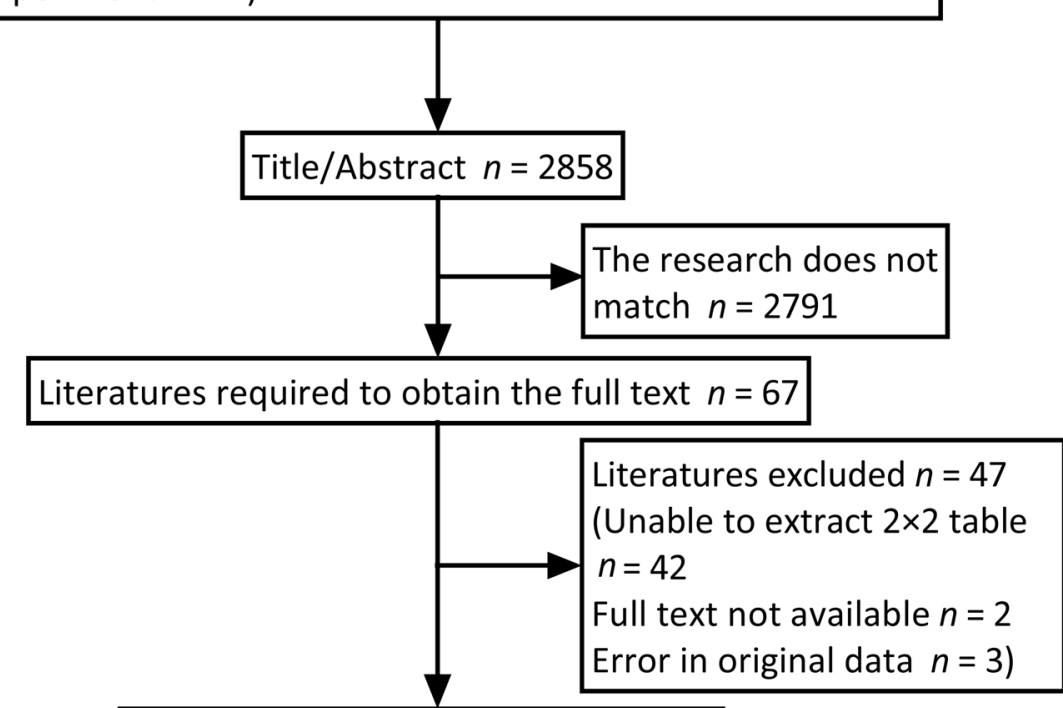

Number of literatures included $n=20$

Fig. 1 Flowchart of literature selection process used for this meta-analysis. CBM (http://sinomed.ac.cn/), CNKI (https://www.cnki.net/), Wan-fang (https://www.wanfangdata.com.cn/index.html), and VIP (http://cstj.cqvip.com/)

Tracing back to the original study revealed that this study was a prospective cohort study of the diagnosis of COVID-19 in emergency patients at the Department of Emergency Surgery, Royal College of Surgeons, UK, and the cases selected may not have been representative of the general population. Other original studies did not make the results sensitive [21-33, 35-40]. In general, this study features stable results, and AUROC is highly accurate.

It can be clearly seen from the results in Fig. 6e that $P=0.57$, which indicates that the funnel plot is symmetrical. Therefore, it can be determined that there is no publication bias in this study, and the conclusions of this study are accurate and reliable.

\section{Discussion}

To investigate the value of $\mathrm{CT}$ imaging for the diagnosis of COVID-19, 20 diagnostic studies were included in this study. The results prove that the diagnostic value of $\mathrm{CT}$ is close to the reference standard. At present, there is disagreement about the role of CT in the diagnosis of COVID-19. Some scholars believed that [18] the chest $\mathrm{CT}$, which served as the primary screening or diagnostic 
Table 1 Basic characteristics of relevant included literatures

\begin{tabular}{|c|c|c|c|c|c|c|c|c|c|c|}
\hline No & Country & Research time & Published year & Age, years & Gender $\mathrm{M} / \mathrm{F}^{* * *}$ & $\mathrm{TP}^{*}$ & $\mathrm{FP}^{*}$ & $\mathrm{FN}^{*}$ & $\mathrm{TN}^{*}$ & References \\
\hline 1 & China & 6 Jan-6 Feb, 2020 & 2020 & $51 \pm 15$ & $467 / 547$ & 580 & 308 & 21 & 105 & [21] \\
\hline 2 & Brazil & Feb-Mar, 2020 & 2020 & $19-88$ & $N A^{* *}$ & 16 & 10 & 9 & 56 & {$[22]$} \\
\hline 3 & Italy & 27 Feb-4 Jul, 2020 & 2020 & $59 \pm 15.8$ & $288 / 408$ & 520 & 61 & 31 & 84 & [23] \\
\hline 4 & Italy & 4 Mar-19 Mar, 2020 & 2020 & $57 \pm 17$ & $83 / 75$ & 60 & 42 & 2 & 54 & [24] \\
\hline 5 & China & 25 Jan-2 Feb, 2020 & 2020 & $51.3 \pm 17.1$ & 290/297 & 423 & 71 & 10 & 83 & {$[25]$} \\
\hline 6 & French & 3 Mar-4 Apr, 2020 & 2020 & $66.4 \pm 18.6$ & $363 / 331$ & 259 & 49 & 28 & 358 & {$[26]$} \\
\hline 7 & Italy & 3 Mar-9 Apr, 2020 & 2020 & $62.4 \pm 18.2$ & $424 / 349$ & 419 & 66 & 43 & 245 & {$[27]$} \\
\hline 8 & Netherlands & 13 Mar-24 Mar, 2020 & 2020 & $66(55-76)$ & $113 / 80$ & 74 & 35 & 9 & 75 & [28] \\
\hline 9 & Brazil & 1 Mar-14 Apr, 2020 & 2020 & $57.9 \pm 18.0$ & $88 / 71$ & 76 & 4 & 10 & 69 & [29] \\
\hline 10 & France & 1 Mar-28 Mar, 2020 & 2020 & $36-75$ & $N A^{* *}$ & 105 & 8 & 24 & 77 & {$[30]$} \\
\hline 11 & Netherlands & 27 Mar-20 Apr, 2020 & 2020 & $N A^{* *}$ & $157 / 162$ & 120 & 22 & 13 & 164 & {$[31]$} \\
\hline 12 & China & 13 Jan-31 Jan, 2020 & 2021 & $36.9 \pm 14.5$ & $113 / 67$ & 30 & 15 & 4 & 131 & [32] \\
\hline 13 & China & 20 Jan-10 Feb, 2020 & 2020 & $50.7 \pm 17.1$ & $49 / 71$ & 88 & 16 & 3 & 13 & [33] \\
\hline 14 & United Kingdom & 23 Mar-15 May, 2020 & 2020 & $N A^{* *}$ & $N A^{* *}$ & 40 & 37 & 29 & 101 & [34] \\
\hline 15 & France & 6 Mar-22 Apr, 2020 & 2020 & $N A^{* *}$ & $N A^{* *}$ & 919 & 148 & 172 & 955 & [35] \\
\hline 16 & India & 15 Feb-30 Jun, 2020 & 2021 & $62(44-78)$ & $214 / 134$ & 206 & 37 & 11 & 94 & {$[36]$} \\
\hline 17 & France & 18 Mar-24 Apr, 2020 & 2021 & $69 \pm 20$ & $257 / 230$ & 69 & 60 & 10 & 348 & {$[37]$} \\
\hline 18 & China & 20 Jan-5 Mar, 2020 & 2020 & $21-83$ & $87 / 77$ & 73 & 70 & 3 & 18 & {$[38]$} \\
\hline 19 & China & 1 Feb-7 Feb, 2020 & 2020 & $59.4 \pm 0.9$ & $105 / 137$ & 132 & 56 & 15 & 39 & [39] \\
\hline 20 & China & 20 Jan-15 Feb, 2020 & 2020 & $8-82$ & $96 / 68$ & 75 & 55 & 4 & 30 & {$[40]$} \\
\hline
\end{tabular}

*TP true positive, $F P$ false positive, $F N$ false negative, $T N$ true negative; ${ }^{* *} N A$ not available; ${ }^{* *} \mathrm{M}=$ male, $\mathrm{F}=$ female

method, had a high false positive rate in areas with low incidences of COVID-19. Others have suggested that [17] chest CT scans could be used as a primary screening and diagnostic tool for COVID-19. COVID-19 Diagnosis and Treatment Protocol (Trial 5th Revised Version) [41] indicated that suspected cases possessing imaging features of pneumonia in Hubei Province as being clinically diagnosed COVID-19. In addition, the progress of COVID-19 is still unclear, and the continuous emergence of mutant strains has puzzled researchers. CT provides macroscopic imaging changes that are not affected by viral mutations, advances in $\mathrm{CT}$ thin-slice and reconstruction techniques have increased the detection rate of the disease. At present, asymptomatic infection is increasing and the harm is huge. Chen et al. [42] showed that chest CT was helpful for early detection of asymptomatic infected persons. At the same time, CT provides a visual image representation for the follow-up comparison of diseases. It is still necessary to pay attention to the amount of radiation from CT. Fortunately, it has been reported on the study that the use of isocenter can ensure the same image quality and reduce the radiation of $\mathrm{CT}$ [43]. In addition, infection prevention and control management of $\mathrm{CT}$ examination rooms is critical.

The results of this study indicate that the heterogeneity is caused by the threshold effect. Combined with the quality evaluation results of QUADAS-2 in this study, there are high risk of bias and applicability concerns in the flow and timing, patient selection. Analyzing the causes of heterogeneity, the authors believe that it depends on the disease prevalence, the characteristics of the study population, diagnostic criteria, etc. In this study, we conducted a CT screening examination of COVID-19 based on the patient population in the emergency department and fever clinic of various hospitals during the epidemic period, which also included some hospitals that isolated and treated suspected patients, so there was a certain patient selection bias. In the included studies, the guidelines or consensus for CT diagnosis of COVID-19 were inconsistent, as were the qualifications and specialty of the diagnosing physician. All these may be the reasons for the threshold effect (heterogeneity) in this study.

The COVID-19 pathogen is affected by various factors and has a low positive detection rate. This indicated that nucleic acid test may produce false negative results [44, 45]. Among the studies, RT-PCR tests have been repeated on patients with negative results, which can reduce the probability of a false negative to a certain extent. We defined positive results and all negative results after the first or multiple RT-PCR tests as COVID-19 positive and COVID-19 negative, respectively. In particularly, multiple RT-PCR tests are necessary for highly suspected patients with negative RT-PCR. The bias of the reference 


\begin{tabular}{|l|lllll|}
\hline $\begin{array}{r}\text { Patient selection } \\
\text { Index test }\end{array}$ \\
Reference standard \\
Flow and timing
\end{tabular}

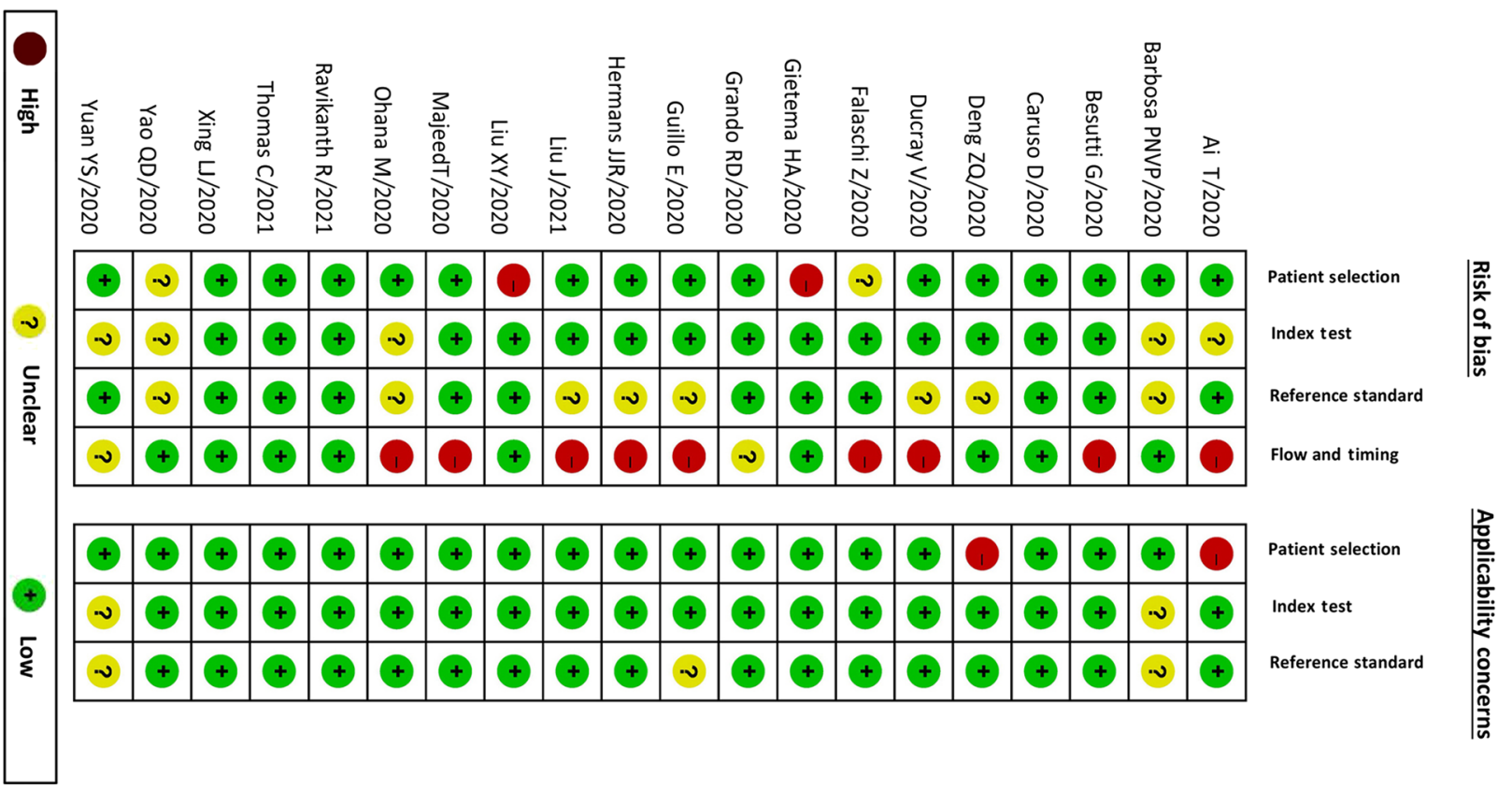

Fig. 2 Risk of bias and applicability concerns graph, risk of bias and applicability concerns summary of 20 included literatures using the Quality Assessment of Diagnostic Accuracy Studies-2 (QUADAS-2) tool
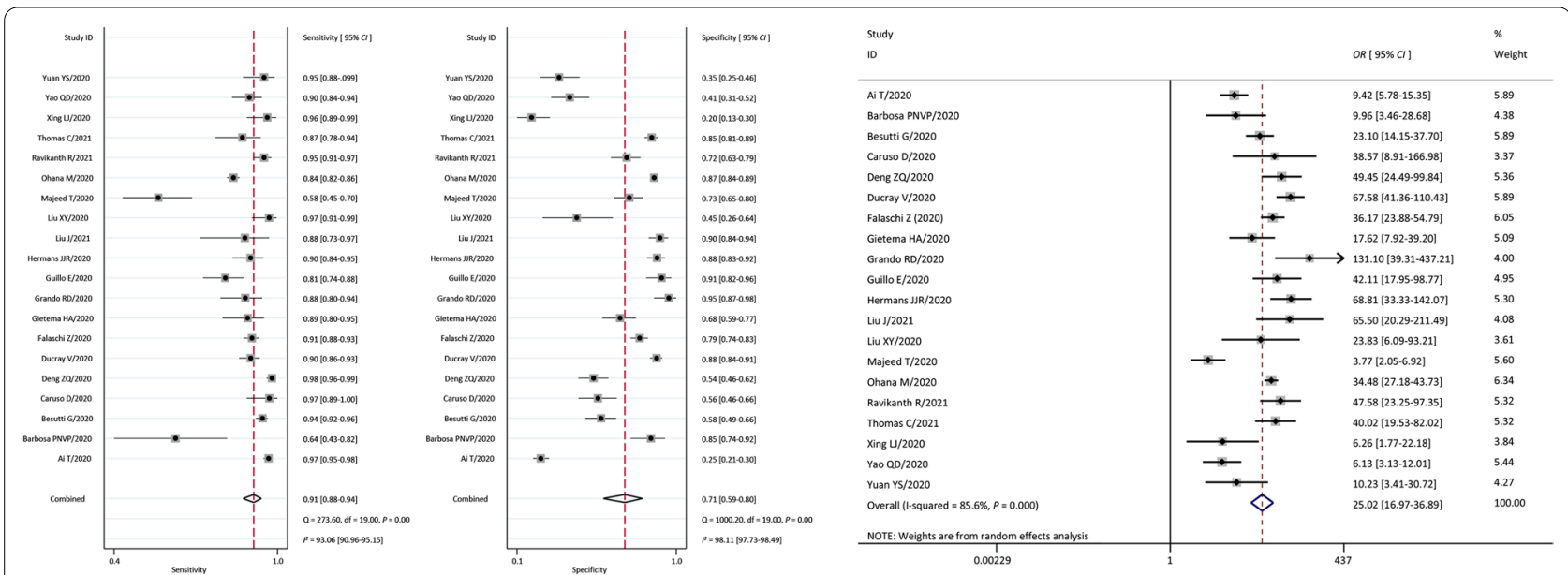

Fig. 3 Coupled forest plots of pooled sensitivity, specificity and OR of Chest CT. Numbers were pooled with 95\% Cl. Corresponding heterogeneity statistics were provided at the bottom. OR odds ratio, Cl confidence interval 


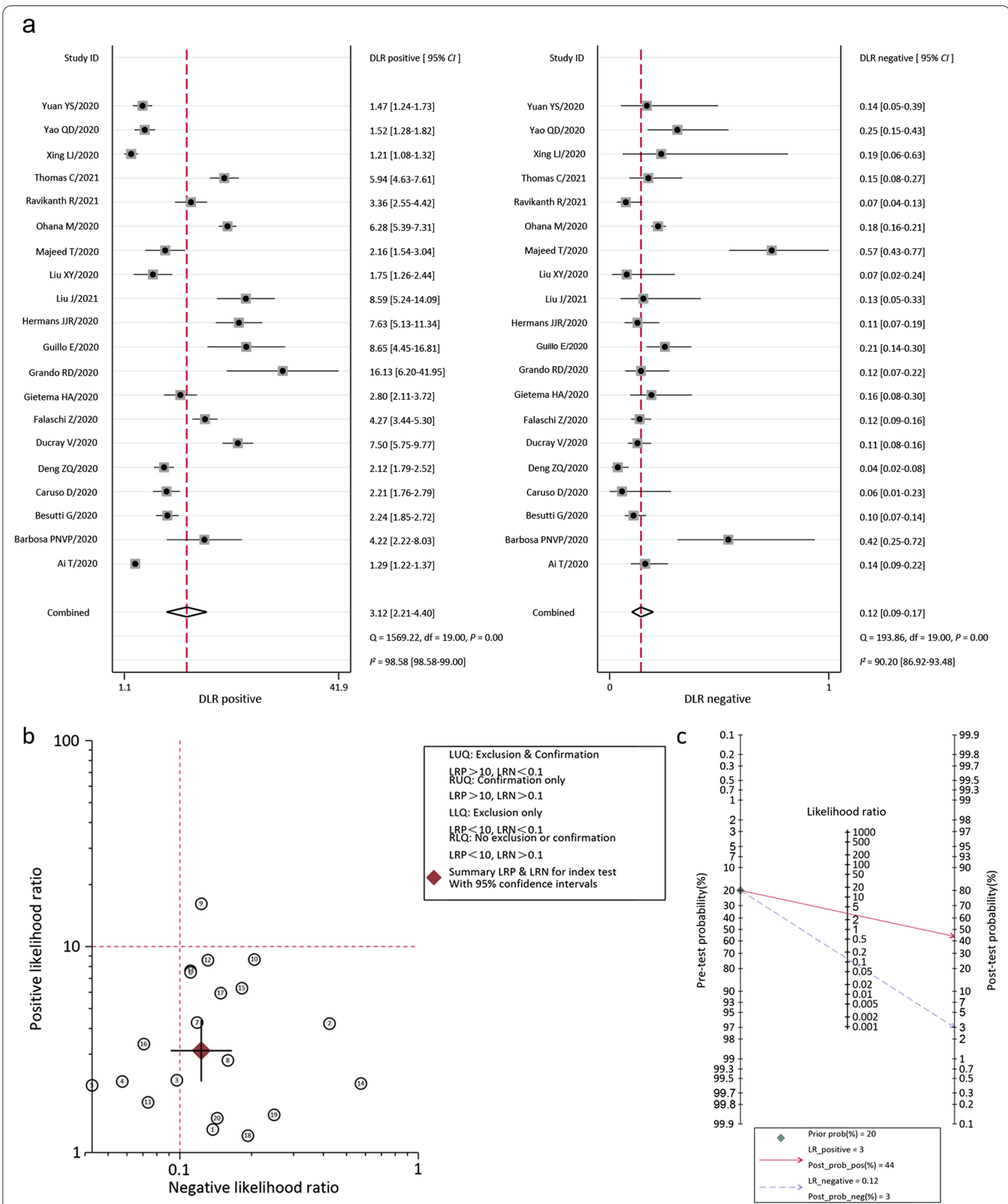

Fig. 4 Coupled forest plots of pooled LR positive and LR negative of chest CT. Numbers were pooled with 95\% Cl (a). A likelihood ratio matrix diagram which summary LR positive and LR negative for index test with $95 \% \mathrm{Cl}(\mathbf{b})$. The probability graph shows the relationship between pre-test probability and post-test probability (c). DLR diagnostic likelihood ratio, Cl confidence interval, LR likelihood ratio, CT computed tomography, LRP likelihood ratio positive, $L R N$ likelihood ratio negative, $R \cup Q$ right upper quadrant, $L L Q$ lift lower quadrant, $R L Q$ right lower quadrant 


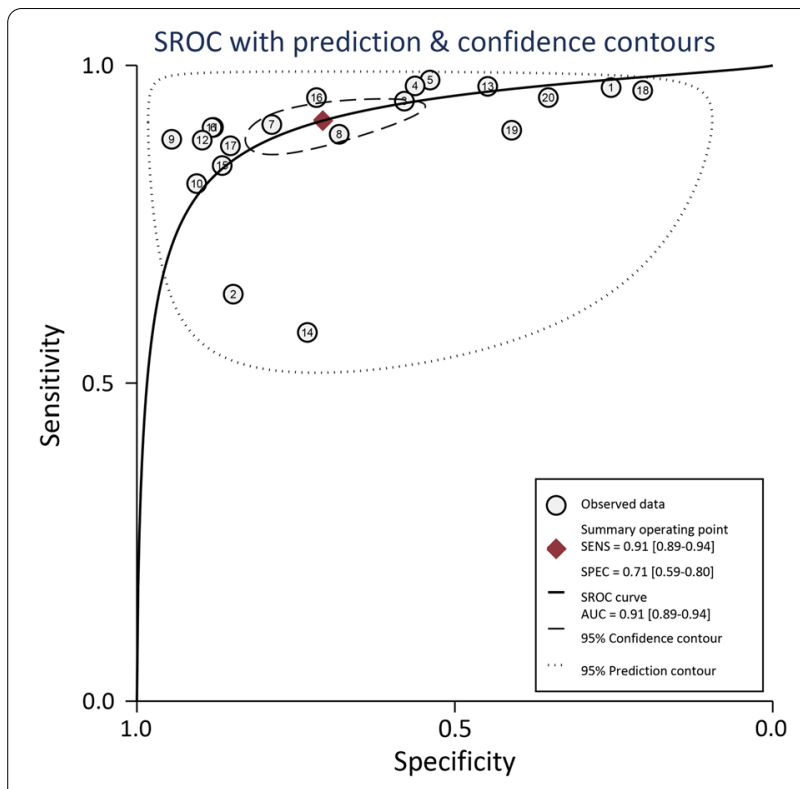

Fig. 5 SROC curve indicated that the area under the curve was 0.91, with the $95 \% \mathrm{Cl}$. SROC summary receiver operating characteristic curves, $\mathrm{Cl}$ confidence interval, SENS sensitivity, AUC area under the curve, SPEC specificity

standard was minimized. The main problem at hand for countries with increasing case numbers is the lack of appropriate screening and diagnostic facilities [46]. A recent summary of clinical observations on COVID-19 in China revealed that some patients had positive RT-PCR later than the onset of the clinical symptoms themselves. However, they had CT imaging changes or related clinical symptoms when RT-PCR tests were negative, and due to the failure to produce a timely diagnosis, some patients progressed rapidly, which affected the prognosis.

It has been more than a year since COVID-19 was first confirmed. Further standardization and unification of CT implementation by experts in related fields to improve the pre-test probability of COVID-19 by front-line physicians will benefit doctors and patients worldwide. At the same time, experts on countries need to cooperate in multiple ways. First, a unified diagnostic standard for CT is significant. Up to now, scholars are still exploring and studying. A meta-analysis on evaluating the diagnostic value of CT using methods such as the COVID-19 Reporting and Data System (CO-RADS) and the Radiological Society of North America (RSNA) Classification System [14]. Smet et al. studied the COVID-19 Reporting and Data System (CO-RADS) diagnostic power of CT [47]. China has also published expert consensus on COVID-19 imaging diagnosis [48]. Then, radiologists need to be professionally trained in these guidelines or consensus. Finally, we should pay attention to standardize the visiting procedures for clinically suspected patients and close contacts by screening the epidemiological history and collecting the history of relevant clinical symptoms, such as fever, cough, myalgia or fatigue, dyspnea, expectoration and chest tightness, diarrhea, nausea or vomiting, abdominal discomfort or pain as well as loss of appetite and olfactory dysgeusia [49]. In conclusion, we should conduct nasopharyngeal/oropharyngeal swab tests for suspected patients and CT examinations for RTPCR-negative patients. Patients suspected of COVID-19 via the initial results of a $\mathrm{CT}$ diagnosis should be isolated and the diagnosis should be further confirmed by multiple RT-PCR tests or specifically RT-PCR tests for lower

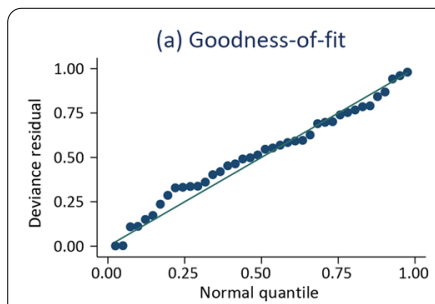

(c) Influence analysis

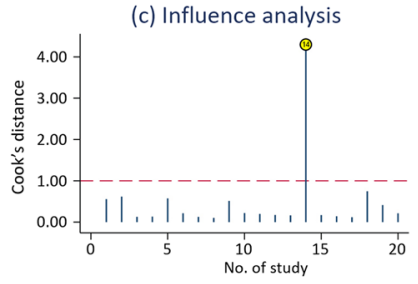

(b) Bivariate normality
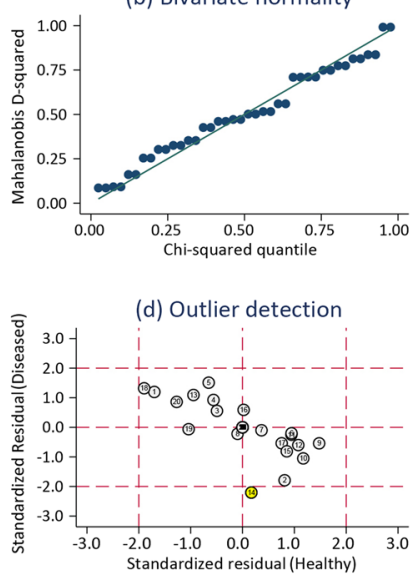

(e)

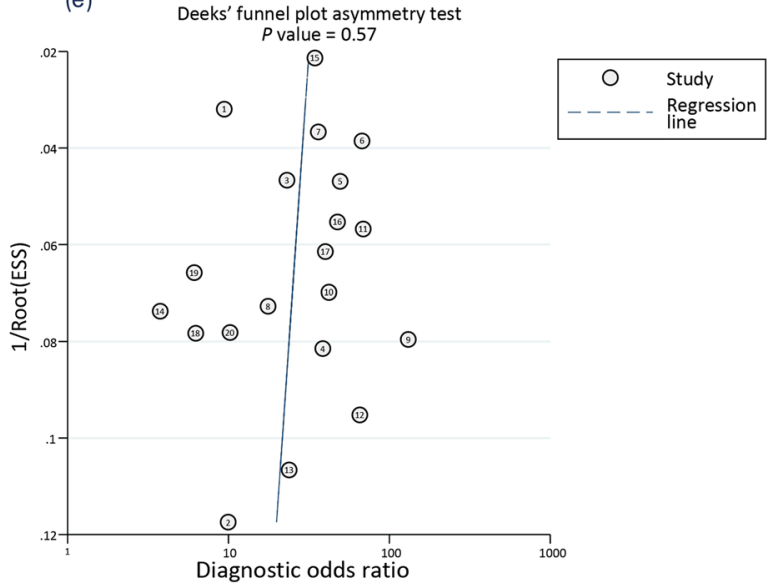

Fig. 6 Sensitivity analysis of four aspects included goodness-of-fit, bivariate normality, influence analysis and qutlier detection (a-d). The publication bias among the included literatures as demonstrated by Deeks'funnel plot (e) 
respiratory tract specimens. Antibody results could also be used to provide further confirmation [45].

There are some limitations of this study. Firstly, there are differences in the regional and medical policies of COVID-19, and the studies we included may not represent the characteristics of the general population for the area in which the study was based. Secondly, most of the studies we included are retrospective studies, and prospective study designs have relatively small sample sizes. Thirdly, the initial inexperience of front-line physicians may have affected the diagnostic performance of $\mathrm{CT}$. Fourthly, given that most of the studies are retrospective, we are concerned about the thoroughness of the blind diagnostic results of CT and RT-PCR. Finally, there may be bias in RT-PCR due to the different reference standards, kits used, sampling methods and target genes detected in each country.

\section{Conclusions}

In this study, we mainly summarized the value of $\mathrm{CT}$ in diagnosing COVID-19, but it also had some limitations. As a rapid and intuitive means of diagnosing chest disease, CT is recommended for rational use for PCR-negative patients with high suspicion of COVID-19. In the event of a global pandemic, the combination of CT and RT-PCR can quickly and accurately confirm a diagnosis and interrupt the transmission chain.

\section{Abbreviations \\ COVID-19: Coronavirus disease 2019; QUADAS-2: Quality assessment of diagnostic studies-2; RT-PCR: Reverse transcriptase-polymerase chain reaction Cl: Confidence interval; DOR: Diagnostic odds ratio; SROC: Summary receiver operating characteristic curve; AUROC: Area under the receiver operating characteristic curve; +LR: Positive likelihood ratio; -LR: Negative likelihood ratio; Sen: Sensitivity; Spe: Specificity; Ac: Accuracy; NPV: Negative predictive value; PPV: Positive predictive value; TP: True positive; FP: False positive; FN: False negative; TN: True negative; CT: Computed tomography.}

\section{Acknowledgements}

The authors would like to thank the authors of all the published research that contributed to the data used in this study.

\section{Authors' contributions}

$J, X Y, J Z L$ and $H J L$ conceived, designed, and executed the literature search. $J L, X Y, Y X Z, Y Z, X T Z$ had access to and analyzed and interpreted the data. All authors have read and approved the manuscript. $\mathrm{HJL}$ and $J \mathrm{~L}$ had final responsibility for the decision to submit for publication.

\section{Funding}

This study was supported by Beijing Municipal Health Commission, technology achievements and appropriate technology promotion project [Grant no. 2020-TG-002]; The National Natural Science Foundation of China [Grant no. 81771806, 61936013]; You'an Medical Development Project of COVID-19 Emergency Prevention and Control Public [Grant no. BJYAYY-2020YC-03]; National Key Research and Development Program of Ministry of Science and Technology [Grant no. SQ2019YFE013267].

\section{Availability of data and materials}

The dataset supporting the conclusion of this article is available from the corresponding author upon reasonable request.

\section{Declarations}

Ethical approval and consent to participate

Not applicable.

\section{Consent for publication}

Not applicable.

\section{Competing interests}

The authors declare that there are no competing interests.

\section{Author details}

${ }^{1}$ Department of Radiology, The Affiliated Infectious Diseases Hospital of Soochow University, The Fifth People's Hospital of Suzhou, Suzhou 215000, Jiangsu, People's Republic of China. ${ }^{2}$ Department of Radiology, Beijing Youan Hospital Capital Medical University, Beijing 100069, People's Republic of China. ${ }^{3}$ Department of Radiology, The First Hospital of Tsinghua University, Beijing 100016, People's Republic of China. ${ }^{4}$ Center for Evidence-Based and Translational Medicine, Zhongnan Hospital of Wuhan University, Wuhan 430071 Hubei, People's Republic of China.

Received: 5 July 2021 Accepted: 8 October 2021

Published online: 21 October 2021

\section{References}

1. Qureshi H. The digestive system and the COVID-19. J Pak Med Assoc. 2020;70(Suppl 3):S98-100.

2. Su S, Shen J, Zhu L, Zhu L, Qiu Y, He JS, et al. Involvement of digestive system in COVID-19: manifestations, pathology, management and challenges. Therap Adv Gastroenterol. 2020;13:1-12.

3. Segura PS, Lázaro YA, Tapia SM, Chaves TC, Domingo JJS. Involvement of the digestive system in COVID-19. a review. Gastroenterol Hepatol. 2020:43:464-71.

4. Noh M. Brain imaging findings in COVID-19: what do we know so far? J Neuroradiol. 2020;47(5):329-30.

5. Tsivgoulis G, Palaiodimou L, Katsanos AH, Caso V, Köhrmann M, Molina C, et al. Neurological manifestations and implications of COVID-19 pandemic. Ther Adv Neurol Disord. 2020;13:1-14.

6. Zhou Y, Ren Q, Chen G, Jin Q, Cui Q, Luo H, et al. Chronic kidney diseases and acute kidney injury in patients with COVID-19: evidence from a meta-analysis. Front Med. 2020;7:588301.

7. Szmuda T, Ali S, Hetzger TV, Rosvall P, Słoniewski P. Are online searches for the novel coronavirus (COVID-19) related to media or epidemiology? A cross-sectional study. Int J Infect Dis. 2020;97:386-90.

8. Li XQ, Cai WF, Huang LF, Chen C, Liu YF, Zhang ZB, et al. Comparison of epidemic characteristics between SARS in 2003 and COVID-19 in 2020 in Guangzhou. Chin J Epidemiol. 2020;41:634-7 (in Chinese).

9. Ali I, Alharbi OML. COVID-19: disease, management, treatment, and social impact. Sci Total Environ. 2020;728:138861.

10. Tahamtan A, Ardebili A. Real-time RT-PCR in COVID-19 detection: issues affecting the results. Expert Rev Mol Diagn. 2020;20(5):453-4.

11. Shi F, Wang J, Shi J, Wu Z, Wang Q, Tang Z, et al. Review of artificial intelligence techniques in imaging data acquisition, segmentation, and diagnosis for COVID-19. IEEE Rev Biomed Eng. 2021;14:4-15.

12. Hernández-Huerta MT, Mayoral LPT, Navarro LMS, Mayoral-Andrade G, Pérez-Campos Mayoral E, Zenteno E, et al. Should RT-PCR be considered a gold standard in the diagnosis of COVID-19. J Med Virol. 2020;92(11):2312-3.

13. Fang $Y$, Zhang $H$, Xie J, Lin M, Ying L, Pang $P$, Ji W. Sensitivity of chest CT for COVID-19: comparison to RT-PCR. Radiology. 2020;296(2):E115-7. 
14. Kwee RM, Adams HJA, Kwee TC. Diagnostic performance of CO-RADS and the RSNA classification system in evaluating COVID-19 at chest CT: a meta-analysis. Radiol Cardiothorac Imaging. 2021;3(1):e200510.

15. Leung K, Shum MH, Leung GM, Lam TT, Wu JT. Early transmissibility assessment of the N501Y mutant strains of SARS-CoV-2 in the United Kingdom, October to November 2020. Euro Surveill. 2021;26(1):2002106.

16. Feng W, Newbigging AM, Le C, Pang B, Peng H, Cao Y, et al. Molecular diagnosis of COVID-19: challenges and research needs. Anal Chem. 2020:92(15):10196-209.

17. Brun AL, Gence-Breney A, Trichereau J, Ballester MC, Vasse M, Chabi ML, et al. COVID-19 pneumonia: high diagnostic accuracy of chest CT in patients with intermediate clinical probability. Eur Radiol. 2021;31(4):1969-77.

18. Kim H, Hong H, Yoon SH. Diagnostic performance of $\mathrm{CT}$ and reverse transcriptase polymerase chain reaction for coronavirus disease 2019: a meta-analysis. Radiology. 2020;296(3):201343.

19. Lieveld AWE, Azijli K, Teunissen BP, van Haaften RM, Kootte RS, van den Berk IAH, et al. Chest CT in COVID-19 at the ED: validation of the COVID19 reporting and data system (CO-RADS) and CT severity score: a prospective, multicenter, observational study. Chest. 2021;159(3):1126-35.

20. Wu L, Zhang Y, Zeng XT. The QUADAS-2 tool for the quality assessment of diagnostic accuracy study: an introduction. J Hubei Univ Med. 2013;32:201-8 (in Chinese).

21. Ai T, Yang Z, Hou H, Zhan C, Chen C, Lv W, et al. Correlation of chest CT and RT-PCR testing for coronavirus disease 2019 (COVID-19) in China: a report of 1014 cases. Radiology. 2020;296(2):E32-32E40.

22. Barbosa PNVP, Bitencourt AGV, de Miranda GD, Almeida MFA, Chojniak R. Chest CT accuracy in the diagnosis of SARS-CoV-2 infection: initial experience in a cancer center. Radiol Bras. 2020;53(4):211-5.

23. Besutti G, Giorgi Rossi P, lotti V, Spaggiari L, Bonacini R, Nitrosi A, et al. Accuracy of $\mathrm{CT}$ in a cohort of symptomatic patients with suspected COVID-19 pneumonia during the outbreak peak in Italy. Eur Radiol. 2020;30(12):6818-27.

24. Caruso D, Zerunian M, Polici M, Pucciarelli F, Polidori T, Rucci C, et al. Chest CT features of COVID-19 in Rome, Italy. Radiology. 2020;296(2):E79-79E85.

25. Deng ZQ, Zhang XC, Li YR, Xu HB, Gang YD, Wang HL, et al. Value of chest CT screening in the early COVID-19 outbreak. Chin J Radiol. 2020;54(5):430-4 (in Chinese).

26. Ducray V, Vlachomitrou AS, Bouscambert-Duchamp M, Si-Mohamed S, Gouttard S, Mansuy A, et al. Chest CT for rapid triage of patients in multiple emergency departments during COVID-19 epidemic: experience report from a large French university hospital. Eur Radiol. 2021;31(2):795-803

27. Falaschi Z, Danna P, Arioli R, Pasché A, Zagaria D, Percivale I, et al. Chest CT accuracy in diagnosing COVID-19 during the peak of the Italian epidemic: a retrospective correlation with RT-PCR testing and analysis of discordant cases. Eur J Radiol. 2020;130:109192.

28. Gietema HA, Zelis N, Nobel JM, Lambriks LJG, van Alphen LB, Oude Lashof AML, et al. CT in relation to RT-PCR in diagnosing COVID-19 in The Netherlands: a prospective study. PLoS One. 2020;15(7):e0235844.

29. Grando RD, Brentano VB, Zanardo AP, Hertz FT, Júnior LCA, Prietto Dos Santos JF, et al. Clinical usefulness of tomographic standards for COVID-19 pneumonia diagnosis: experience from a Brazilian reference center. Braz J Infect Dis. 2020;24(6):524-33.

30. Guillo E, Bedmar Gomez I, Dangeard S, Bennani S, Saab I, Tordjman M, et al. COVID-19 pneumonia: diagnostic and prognostic role of CT based on a retrospective analysis of 214 consecutive patients from Paris, France. Eur J Radiol. 2020;131:109209.

31. Hermans JJR, Groen J, Zwets E, Boxma-De Klerk BM, Van Werkhoven JM, Ong DSY, et al. Chest CT for triage during COVID-19 on the emergency department: myth or truth. Emerg Radiol. 2020;27(6):641-51.
32. Liu J, Chen X, Zhu Y, Liu R, Zhang H, Li M, et al. Value of diagnosis and preliminary screening of COVID-19 in Suzhou area using CT. J Pract Radiol. 2021;37(3):381-4 (in Chinese).

33. Liu $X Y$, Xie $Y L$, Zhang SD, Jiang YP, Wang $X$. The diagnostic value of chest CT scan in the diagnosis of pneumonia caused by 2019-novel coronavirus. Radiol Practe. 2020;35(4):433-6 (in Chinese).

34. Majeed T, Ali RS, Solomon J, Mesri M, Sharma S, Shamim S, et al. The role of the computed tomography (CT) thorax in the diagnosis of COVID-19 for patients presenting with acute surgical emergencies. A single institute experience. Indian J Surg. 2020;1(6):1-635.

35. Ohana M, Muller J, Severac F, Bilbault P, Behr M, Oberlin M, et al. Temporal variations in the diagnostic performance of chest CT for Covid-19 depending on disease prevalence: experience from North-Eastern France. Eur J Radiol. 2020;134:109425.

36. Ravikanth R. Diagnostic accuracy of chest computed tomography in improving the false negative rate as compared to reverse transcriptase polymerase chain reaction in coronavirus disease 2019 pneumonia: a cross sectional analysis of 348 cases from India. Lung India. 2021;38:S11-21.

37. Thomas C, Naudin M, Tasu JP, Leclerc C, Depaire L, Subervillle M, et al. Efficacy of chest CT scan for COVID-19 diagnosis in a low prevalence and incidence region. Eur Radiol. 2021;1-6.

38. Xing LJ, Zhang RF, Chai YR. Assessment of CT findings and nucleic acid testing in the diagnosis and the disease course of COVID-19: a comparative study. Int J Radiat Med Nucl Med. 2020:44(10):610-539(in Chinese).

39. Yao QD, Zhang CB, Fu J, Zhang DY, Liu HF. Manifestation and diagnostic value of Chest CT in patients with COVID-19. Jiangsu Med. 2020;46(6):564-7 (in Chinese).

40. Yuan YS, Ma PQ, Zhang L, Zhang Y, Peng B, Qiao AL, et al. Clinical analysis on clinical value of initial chest CT findings in patients with COVID-19. J Wannan Med Coll. 2020:39(6):573-6 (in Chinese).

41. National Health Commission. COVID-19 Diagnosis and Treatment Protocol (Trial 5th Revised Version). CJITWM. 2020; 40:136-8. (in Chinese)

42. Chen C, Zhu C, Yan D, Liu H, Li D, Zhou Y, et al. The epidemiological and radiographical characteristics of asymptomatic infections with the novel coronavirus (COVID-19): a systematic review and meta-analysis. Int J Infect Dis. 2021;104:458-64

43. Booij R, Budde R, Dijkshoorn ML, van Straten M. Accuracy of automated patient positioning in CT using a 3D camera for body contour detection. Eur Radiol. 2019;29(4):2079-88.

44. Lu R, Zhao X, Li J, Niu P, Yang B, Wu H, et al. Genomic characterisation and epidemiology of 2019 novel coronavirus: implications for virus origins and receptor binding. Lancet. 2020;395(10224):565-74.

45. Zhong L, Chuan J, Gong B, Shuai P, Zhou Y, Zhang Y, et al. Detection of serum IgM and IgG for COVID-19 diagnosis. Sci China Life Sci. 2020;63(5):777-80.

46. Nepal R, Bhattarai B. The grim reality of health system uncovered with COVID-19 pandemic in Nepal. J Nepal Health Res Counc. 2020;18(3):569-71.

47. Smet KD, Smet DD, Ryckaert T, Laridon E, Heremans B, Vandenbulcke $\mathrm{R}$, et al. Diagnostic performance of chest CT for SARS-CoV-2 Infection in individuals with or without COVID-19 symptoms. Radiology. 2021:298(1):E30-7.

48. Yang Q, Liu Q, Xu H, Lu H, Liu S, Li H. Imaging of coronavirus disease 2019: a Chinese expert consensus statement. Eur J Radiol. 2020;127:109008.

49. Jin YH, Zhan QY, Peng ZY, Ren XQ, Yin XT, Cai L, et al. Chemoprophylaxis, diagnosis, treatments, and discharge management of COVID19: an evidence based clinical practice guideline. Med J Chin PLA. 2020;45(10):1003-31 (in Chinese) 